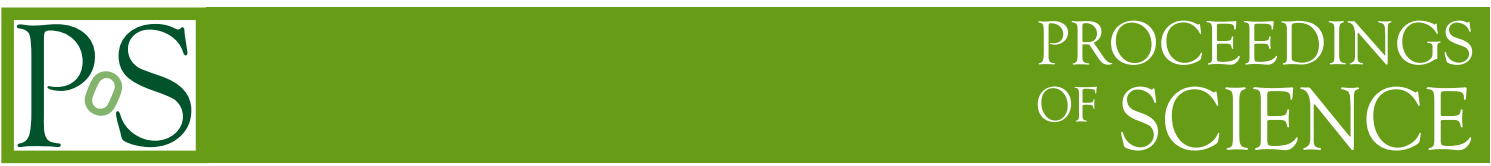

\title{
Revealing jet radio emission from intermediate-mass black holes
}

\author{
Mar Mezcua* \\ ${ }^{1}$ Harvard-Smithsonian Center for Astrophysics (CfA), 60 Garden Street, Cambridge, \\ Massachusetts 02138, USA \\ ${ }^{2}$ Instituto de Astrofísica de Canarias (IAC), E-38200 La Laguna, Tenerife, Spain \\ ${ }^{3}$ Universidad de La Laguna, Dept. Astrofísica, E-38206 La Laguna, Tenerife, Spain \\ E-mail: mar.mezcuadefa.harvard.edu
}

\begin{abstract}
Relativistic jets were first discovered in the nuclei of galaxies. The later finding of collimated jets arising from the compact counterpart of X-ray binaries suggested that jets should be present not only in supermassive and stellar-mass black holes, but also in intermediate-mass black holes (IMBHs). According to evolutionary models of black hole growth, IMBHs could be the initial seed of supermassive black holes. They could form from very young and massive stars or from the direct collapse of pre-galactic gas discs. These processes should have left a population of IMBHs in the haloes of galaxies, where they could be observed as ultraluminous X-ray sources (ULXs). However, observational evidence of IMBHs and of their jet radio emission is scarce.

I will review the few detected IMBHs with jet radio emission and present the results of European VLBI Network (EVN) observations aimed at studying radio emission in ULXs and clarifying the nature of these sources. The EVN observations revealed compact radio emission from the ULX N5457-X9, which becomes a potential IMBH candidate, as well as the detection of pc-scale jet emission from an IMBH in the spiral arm of NGC 2276. With a total radio lobe size of $\sim 650 \mathrm{pc}$, this source could be the largest non-nuclear extragalactic jet ever discovered.
\end{abstract}

12th European VLBI Network Symposium and Users Meeting

7-10 October 2014

Cagliari, Italy

${ }^{*}$ Speaker. 


\section{Introduction}

The largest population of black holes for which extensive observational evidence exists is that formed by supermassive black holes (SMBHs; $M_{\mathrm{BH}}>10^{6} \mathrm{M}_{\odot}$ ). SMBHs are thought to be ubiquitous in the centers of all massive galaxies (e.g., Kormendy \& Richstone 1995; Kormendy \& Ho 2013; McConnell \& Ma 2013), although evidence is growing that some may also reside in the nucleus of low-mass dwarf galaxies (e.g., Reines et al. 2011, 2014; Seth et al. 2014). On the other hand, several tens of stellar-mass BHs $\left(M_{\mathrm{BH}}<20 \mathrm{M}_{\odot}\right)$ have been found within our Galaxy and beyond (e.g., Remillard \& McClintock 2006). While stellar-mass BHs are thought to result from the death of high-mass stars, the mechanisms responsible for the formation and growth of SMBHs are still unclear. The finding that SMBHs already existed only 800 Myr after the Big Bang (e.g., Mortlock et al. 2011) suggests that they had to grow from lower-mass seed BHs via mergers or super-Eddington accretion (e.g., Volonteri 2010; Volonteri \& Silk 2014; Alexander \& Natarajan 2014). These seed or intermediate-mass BHs (IMBHs), with masses $100<M_{\mathrm{BH}}<10^{6} \mathrm{M}_{\odot}$, would bridge the gap between stellar-mass and SMBHs.

The strong correlations found between the mass of SMBHs and their host galaxy properties (like the luminosity, bulge mass, and stellar velocity dispersion, e.g., Magorrian et al. 1998; Ferrarese \& Merritt 2000; Kormendy \& Richstone 1995; Kormendy \& Ho 2013; McConnell \& Ma 2013) indicate that the least massive galaxies, i.e. dwarf galaxies, may harbor BHs of intermediate masses similar to those seed IMBHs from the early Universe (Bellovary et al. 2011). IMBHs could form from direct collapse of gas (Lodato \& Natarajan 2006), from the death of Population III stars (Belczynski et al. 2010), or from runaway stellar mergers in dense stellar clusters (Portegies Zwart et al. 2004). Therefore, they should be present not only in the nucleus of dwarf galaxies but also in globular clusters, in dense regions of star formation in spiral galaxies, and in the halos of galaxies having undergone a minor merger event that stripped the low-mass satellite galaxy. Alternatively, IMBHs could efficiently form in the disks of active galactic nuclei (AGN) by the merging of nuclear stellar and compact objects (McKernan et al. 2011).

Finding proof of the existence of IMBHs has important implications not only for studies of SMBH and galaxy growth but also for the epoch of reionization (e.g., Ricotti \& Ostriker 2004; Johnson et al. 2014), the detection of gravitational waves (e.g., Hughes 2002; Abbott et al. 2009), for investigating whether BH feedback plays a role in galaxy formation (i.e. by suppressing star formation) at all mass scales (e.g., Kim et al. 2011; Dubois et al. 2012), and for testing whether the accretion physics is scale invariant (e.g., Nemmen et al. 2012; Gultekin et al. 2014). Despite their importance, observational evidence of IMBHs still remains elusive.

\section{Searching for observational evidence}

\subsection{Nuclear IMBHs}

Bulgeless, low-mass, and dwarf galaxies have been the focus of many IMBH searches. The most reliable way for weighting BHs is to use the motion of gas and stars within the BH radius of influence. However, measuring $\mathrm{BH}$ masses in the intermediate regime by means of stellar or gas dynamics has been possible only for galaxies out to $\sim 3 \mathrm{Mpc}\left(\mathrm{NGC} 404 ; M_{\mathrm{BH}} \sim 5 \times 10^{5} \mathrm{M}_{\odot}\right.$; Seth et al. 2010), while upper limits have been obtained for several galaxies in the Local Group 
(NGC 205, Ursa Minor, Fornax, $M_{\mathrm{BH}} \leq$ a few $\times 10^{4} \mathrm{M}_{\odot}$, Valluri et al. 2005, Lora et al. 2009, Jardel \& Gebhardt 2012; M33, $M_{\mathrm{BH}} \leq$ a few $\times 10^{3} \mathrm{M}_{\odot}$, Gebhardt et al. 2001) and out to distances of $\sim 24 \mathrm{Mpc}$ (NGC 2139; $M_{\mathrm{BH}}<1.5 \times 10^{5} \mathrm{M}_{\odot}$; Neumayer \& Walcher 2012).

The alternative is to use less reliable methods that are based on the radiative signatures of the accreting $\mathrm{BH}$, such as reverberation mapping. This is the case for the IMBHs in the dwarf galaxies NGC 4395 and POX 52, which are the first AGN for which a BH mass in the intermediate regime $\left(\sim 3 \times 10^{5} \mathrm{M}_{\odot}\right.$; Peterson et al. 2005; Thornton et al. 2008) was obtained from the width of optical/UV broad emission lines. Later systematic searches for low-mass BHs using optical broad line emission yielded the detection of a few hundred candidates with $M_{\mathrm{BH}} \sim 10^{5}-10^{6} \mathrm{M}_{\odot}$ (Greene \& Ho 2004; Greene \& Ho 2007; Dong et al. 2007, 2012; Reines et al. 2013). These optical samples are usually biased toward type 1 AGN, while the virial mass estimates fail to detect BHs with masses below $\sim 10^{5} \mathrm{M}_{\odot}$ (Reines et al. 2013); hence, further studies have focused on the detection of IMBHs based on mid-infrared spectroscopy (e.g., Satyapal et al. 2008, 2009), infrared colors (e.g., Marleau et al. 2014) and X-ray observations (e.g., Dewangan et al. 2008; Desroches, Greene \& Ho 2009; Gallo et al. 2010; Terashima et al. 2012; Schramm et al. 2013; Secrest et al. 2013; Yuan et al. 2014). The best confirmation of the presence of an accreting BH in those optical and infrared-selected IMBH candidates comes from the detection of hard, unresolved X-ray emission. If this is spatially coincident with jet-core radio emission, an estimate of the BH mass can be obtained using the fundamental plane of accreting BHs, which is a correlation between radio luminosity, X-ray luminosity and BH mass mass valid from stellar-mass to SMBHs in the low/hard X-ray state (i.e. accreting at sub-Eddington rates; e.g., Merloni et al. 2003; Gültekin et al. 2014 and references therein). In addition, the detection of a radio counterpart allows us to measure the brightness temperature and spectral properties (i.e. radio spectral index) and therefore to assess which is the physical mechanism responsible for the radio emission. However, very few systematic searches for IMBHs have been carried out in the radio regime and in very few of the nuclear IMBH candidates has jet radio emission been detected (NGC 4395, Wrobel \& Ho 2006; GH10, Greene et al. 2006, Wrobel et al. 2008; NGC 404, Nyland et al. 2012). A compilation of IMBHs with radio counterparts is presented in Table 1.

\subsection{Off-nuclear IMBHs}

In the case of off-nuclear IMBHs, some disputed candidates have been suggested in globular clusters based on dynamic mass measurements $\left(M_{\mathrm{BH}} \leq\right.$ a few $\times 10^{4} M_{\odot}$, e.g., Gebhardt et al. 2005; van der Marel \& Anderson 2010; Lützgendorf et al. 2011) and stringent radio upper limits (e.g., Cseh et al. 2010; Wrobel et al. 2011; Strader et al. 2012). The most compelling observational evidence for non-nuclear IMBHs has been found in ultraluminous X-ray sources (ULXs), which are extragalactic, point-like $\mathrm{X}$-ray sources with isotropic $\mathrm{X}$-ray luminosities above the Eddington limit of a $20 M_{\odot}$ stellar-mass BH $\left(L_{\mathrm{X}}>3 \times 10^{39} \mathrm{erg} \mathrm{s}^{-1}\right)$. Although the nature of most ULXs could be explained by stellar-mass BHs accreting at super-Eddington rates (e.g., Begelman 2002; Poutanen et al. 2007; Motch et al. 2014) or by massive stellar BHs (20-80 $\mathrm{M}_{\odot}$; e.g., Mapelli et al. 2009; Zampieri \& Roberts 2009; Cseh et al. 2014), a subset remain as potential IMBHs based on their variability, quasi-periodic oscillations (M82-X1; Pasham et al. 2014), and extremely high X-ray luminosities $\left(L_{X} \geq 10^{41} \mathrm{erg} \mathrm{s}^{-1}\right.$, the so-called Hyperluminous X-ray sources, HLXs; e.g., Farrell et al. 2009; Sutton et al. 2012). The later, supported by the detection of X-ray state 
Table 1: IMBHs with radio emission.

\begin{tabular}{|c|c|c|c|c|c|c|c|c|}
\hline Object & $\begin{array}{c}\text { Galaxy } \\
\text { type } \\
\text { (2) }\end{array}$ & $\begin{array}{c}{[\mathrm{Mpc}]} \\
\text { (3) }\end{array}$ & $\begin{array}{r}M_{\odot} \\
(4)\end{array}$ & $\begin{array}{c}{\left[\mathrm{erg} \mathrm{s}^{-1}\right]} \\
(5)\end{array}$ & $\begin{array}{c}{\left[\operatorname{erg~s}^{-1}\right]} \\
(6)\end{array}$ & $\begin{array}{c}\text { Jet size } \\
\text { [pc] } \\
(7)\end{array}$ & (8) & Ref. \\
\hline \multicolumn{9}{|l|}{ Nuclear } \\
\hline NGC 4395 & $\mathrm{Sdm}$ & 4.3 & $3.6 \times 10^{5}$ & $8.8 \times 10^{39}$ & $7.6 \times 10^{33}$ & 0.3 & -0.6 & $1-3$ \\
\hline GH10 & $\mathrm{dSph}$ & 363 & $8 \times 10^{5}$ & $1.9 \times 10^{42}$ & $6 \times 10^{38}$ & $<320$ & -0.76 & $4-6$ \\
\hline NGC 404 & dS0 & 3.1 & $4.5 \times 10^{5}$ & $1.2 \times 10^{37}$ & $4 \times 10^{34}$ & $\sim 6^{*}$ & -0.88 & $7-8$ \\
\hline \multicolumn{9}{|l|}{ Off-nuclear } \\
\hline HLX-1 & S0/a & 95 & $0.3-30 \times 10^{4}$ & $1.1 \times 10^{42}$ & $1.2 \times 10^{36}$ & $\sim 350$ & -0.4 & $9-12$ \\
\hline NGC2276-3c & $\mathrm{SABc}$ & 33.3 & $\sim 5 \times 10^{4}$ & $1.6 \times 10^{40}$ & $1.4 \times 10^{35}$ & $1.8^{\dagger}$ & -0.5 & $13-14$ \\
\hline
\end{tabular}

Column designation: (1) Object name; (2) galaxy type; (3) luminosity distance; (4) BH mass; (5) peak $\mathrm{X}$-ray luminosity; $(6,7)$ integrated radio luminosity and radio jet size obtained from Karl G. Jansky Very Large Array (VLA) observations at $5 \mathrm{GHz}$ for GH10 and NGC 404, from the VLBI High Sensitivity Array (HSA) at $1.4 \mathrm{GHz}$ for NGC 4395, from the Australia Telescope Compact Array (ATCA) at $5 \mathrm{GHz}$ for HLX-1 (note that the radio emission is transient and can reach $L_{5-9 \mathrm{GHz}}=8 \times 10^{36} \mathrm{erg} \mathrm{s}^{-1}$ ), and from the EVN at 1.6 GHz for NGC2276-3c; (8) radio spectral index, defined as $S \propto v^{\alpha}$; (9) references: [1] Thim et al. 2004, [2] Peterson et al. 2005, [3] Wrobel et al. 2006, [4] Green \& Ho 2004, [5] Barth, Greene \& Ho 2005, [6] Wrobel et al. 2008, [7] Binder et al. 2011, [8] Nyland et al. 2012, [9] Farrell et al. 2009, [10] Davis et al. 2011, [11] Webb et al. 2012, [12] Cseh et al. 2014, [13] Sutton et al. 2012, [14] Mezcua et al. 2015.

* No radio emission was detected for NGC 404 on VLBI scales (Paragi et al. 2014).

${ }^{\dagger}$ Two radio lobes of total size $\sim 650 \mathrm{pc}$ and oriented along the pc-scale jet were detected with the VLA at 5 $\mathrm{GHz}$ (Mezcua et al. 2015).

transitions associated to transient jet radio emission, yielded the discovery of an IMBH of $0.3 \times$ $10^{4}-3 \times 10^{5} M_{\odot}$ in the outskirts of the galaxy ESO 243-49 (HLX-1, e.g., Farrell et al. 2009; Davis et al. 2011; Webb et al. 2012; Cseh et al. 2014). The radio luminosity of HLX-1 in its hard X-ray spectral state lays in the same range of radio luminosities of the nuclear IMBHs $\left(\sim 10^{34}-10^{38} \mathrm{erg}\right.$ $\mathrm{s}^{-1}$, see Table 1); however, no radio emission has so far been detected for most ULXs down to $L_{\mathrm{R}} \sim 10^{34} \mathrm{erg} \mathrm{s}^{-1}$ [e.g., only $11 \mathrm{ULX}$ radio counterparts were found in a cross-match of the Liu \& Bregman 2005 ULX catalog with the FIRST survey (Sánchez-Sutil et al. 2006), 7 when using the ULX catalog of Swartz et al. 2004 (Pérez-Ramírez et al. 2011), and only 1 out of 7 extreme ULXs was detected in the $5 \mathrm{GHz}$ VLA campaign of Mezcua et al. 2013c]. This suggests that most ULXs are not radio supernovae (as these have typical radio luminosities one to three orders of magnitude larger than that of ULXs, e.g., Weiler et al. 2002) nor IMBHs with steady jet radio emission (as their typical radio luminosities are $>10^{34} \mathrm{erg} \mathrm{s}^{-1}$; see Table 1), which reinforces their most likely nature as either super-Eddington accreting stellar-mass BHs, massive stellar-mass BHs, or even neutron stars (Bachetti et al. 2014).

The few detections of a resolved ULX radio counterpart have allowed us to distinguish between extended emission from either jets (e.g., Holmberg II X-1, Cseh et al. 2014; NGC2276-3c, Mezcua et al. 2013c, 2015), nebulae powered by stellar-mass BHs (e.g., NGC5408 X-1, IC 342 


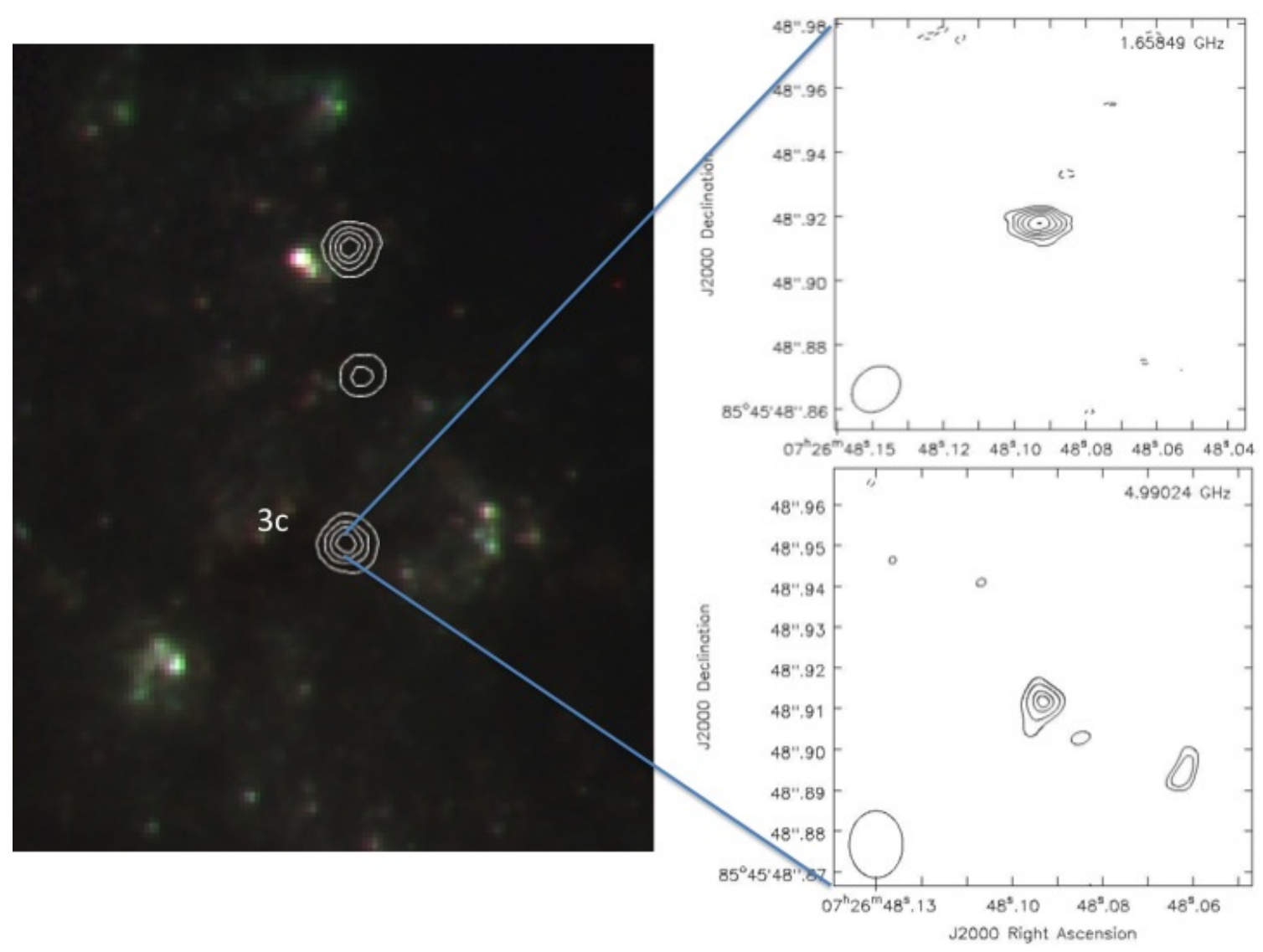

Figure 1: Left: Hubble Space Telescope RGB (red: $\sim 0.8 \mu \mathrm{m}$, green: $\sim 0.6 \mu \mathrm{m}$, blue: $\sim 0.55 \mu \mathrm{m}$ ) image of the western arm of NGC 2276 where the ULX NGC2276-3c is located. The Chandra contours in the 0.2$8 \mathrm{keV}$ band are shown in white. Right: EVN radio maps at $1.6 \mathrm{GHz}$ (top) and $5 \mathrm{GHz}$ (bottom). The $1.6 \mathrm{GHz}$ map has a synthesized beam with a full width at half maximum (FWHM) of $16.4 \times 13.1$ milliarcseconds oriented at a position angle of -52 degrees. Contours are plotted as $[-3,4,5,6,7,8,9]$ times the off-source r.m.s. noise of $8 \mu \mathrm{Jy}_{\text {beam }}{ }^{-1}$. The map at $5 \mathrm{GHz}$ was produced with the same FWHM as the $1.6 \mathrm{GHz}$ radio map. Contours are plotted as $[-5,5,6,7,7.5]$ times the off-source r.m.s. noise of $6 \mu \mathrm{Jy}$ beam $^{-1}$. See Mezcua et al. (2015) for further details.

X-1, Holmberg II X-1, Kaaret et al. 2003, Cseh et al. 2012; MQ1, Soria et al. 2014) or supernova remnants (SNRs; e.g., SNR 4449-1, Mezcua et al. 2013b). In the case of compact radio emission spatially coincident with the X-ray counterpart (e.g., Mezcua \& Lobanov 2011; Mezcua et al. 2013a, 2014b, 2015), the radio luminosity has been used to estimate the ULX BH mass via the fundamental plane of accreting $\mathrm{BHs}$, while the ratio of radio to X-ray luminosity has provided an additional hint on the ULX nature via comparison with the typical ratios obtained for different types of sources (e.g., X-ray binaries, AGN, SNRs; Terashima \& Wilson 2003; see also table 3 in Mezcua et al. 2013a). This revealed the possible presence of an IMBH in the ULX N5457-X9, for which a radio counterpart of $0.12 \mathrm{mJy}\left(\sim 1 \times 10^{34} \mathrm{erg} \mathrm{s}^{-1}\right)$ was detected with the EVN at 1.6 GHz (Mezcua et al. 2013a), and in the extreme ULX NGC2276-3c, for which a radio component of $\sim 10^{35} \mathrm{erg} \mathrm{s}^{-1}$ and size $\sim 1.8 \mathrm{pc}$ has been detected in recent EVN observations at $1.6 \mathrm{GHz}$ and $5 \mathrm{GHz}$ (Fig. 1; Mezcua et al. 2015). The pc-scale radio component of NGC2276-3c has a spectral 
index consistent with flat to optically thin synchrotron emission (see Table 1) and is located in between two peaks of lobe radio emission separated by $\sim 650 \mathrm{pc}$ (Mezcua et al. 2013c). Its position is also spatially coincident with the Chandra X-ray counterpart, whose X-ray spectrum is consistent with the ULX being in the low/hard state at the time of the radio observations. This allows us to estimate the $\mathrm{BH}$ mass using the fundamental plane of accreting BHs, which yields $M_{\mathrm{BH}}=5 \times 10^{4}$ $M_{\odot}$ with a scatter of 0.7 dex (Mezcua et al. 2015) and makes NGC2276-3c the second most compelling off-nuclear IMBH (after HLX-1) with jet radio emission. The location of NGC2276-3c in an unusual spiral arm of the host galaxy (Mezcua et al. 2013c, 2015) and of N5457-X9 in a giant HII region also in the host's spiral arm (Mezcua et al. 2013a) suggest that the putative IMBHs are the nucleus of a tidally stripped dwarf galaxy, as was also suggested for HLX-1 (Farrell et al. 2012, 2014). The nucleus of the stripped satellite galaxy in minor mergers offer thus a new environment in which to look for IMBHs (e.g., Mezcua et al. 2014a).

\section{Conclusions}

The detection of a radio counterpart in those sources candidates to being an IMBH allows us not only to confirm its $\mathrm{BH}$ nature in the case of jet/core radio emission but also to determine the properties of the source and its environment by following a multiwavelength approach. In particular, VLBI radio observations have been proven to be key in resolving out diffuse emission and detecting the IMBH jet/core as well as in estimating the BH mass by means of the fundamental plane of accreting BHs. The advent of deep, very sensitive radio surveys like the Square Kilometre Array (SKA) combined with high-resolution radio observations (e.g., SKA-VLBI) will allow us to detect IMBHs at distances $>100$ Mpc (e.g., Paragi et al. 2014; Wolter et al. 2014) and thus to probe the existence of a population of $\mathrm{BH}$ seeds beyond the nearby Universe.

\section{References}

[1] Abbott, B. P., Abbott, R., Adhikari, R., et al. 2009, Phys. Rev. D., 80, 062001

[2] Alexander, T. \& Natarajan, P. 2014, Science, 345, 1330

[3] Bachetti, M.; Harrison, F. A.; Walton, D. J., et al., 2014, Nature, 514, 202

[4] Barth, A. J., Greene, J. E., \& Ho, L. C. 2005, ApJL, 619, L151

[5] Begelman, M. C. 2002, ApJL, 568, L97

[6] Belczynski, K.; Bulik, T.; Fryer, C. L., et al. 2010, ApJ, 714, 1217

[7] Bellovary, J., Volonteri, M., Governato, F., et al. 2011, ApJ, 742, 13

[8] Binder, B., Williams, B. F., Eracleous, M., et al. 2011, ApJ, 737, 77

[9] Cseh, D., Kaaret, P., Corbel, S., et al. 2010, MNRAS, 406, 1049

[10] Cseh, D.; Corbel, S.; Kaaret, P., et al. 2012, ApJ, 749, 17

[11] Cseh, D.; Kaaret, P.; Corbel, S., et al. 2014, MNRAS, 439, L1

[12] Davis, S. W.; Narayan, R.; Zhu, Y., et al. 2011, ApJ, 734, 111

[13] Desroches, L.-B., Greene, J. E., \& Ho, L. C. 2009, ApJ, 698, 1515 
[14] Dewangan, G. C., Mathur, S., Griffiths, R. E., \& Rao, A. R. 2008, ApJ, 689, 762

[15] Dong, X.; Wang, T.; Yuan, W., et al. 2007, ApJ, 657, 700

[16] Dong, X.-B., Ho, L. C., Yuan, W., et al. 2012, ApJ, 755, 167

[17] Dubois, Y.; Devriendt, J.; Slyz, A. \& Teyssier, R. 2012, MNRAS, 420, 2662

[18] Farrell, S. A.; Webb, N. A.; Barret, D.; Godet, O. \& Rodrigues, J. M. 2009, Nature, 460, 73

[19] Farrell, S. A.; Servillat, M.; Pforr, J., et al. 2012, ApJL, 747, L13

[20] Ferrarese, L. \& Merritt, D. 2000, ApJL, 539, L9

[21] Gallo, E., Treu, T., Marshall, P. J., et al. 2010, ApJ, 714, 25

[22] Gebhardt, K., Lauer, T. R., Kormendy, J., et al. 2001, AJ, 122, 2469

[23] Gebhardt, K., Rich, R. M., \& Ho, L. C. 2005, ApJ, 634, 1093

[24] Greene, J. E. \& Ho, L. C. 2004, ApJ, 610, 722

[25] Greene, J. E. \& Ho, L. C. 2007, ApJ, 670, 92

[26] Greene, J. E., Ho, L. C., \& Ulvestad, J. S. 2006, ApJ, 636, 56

[27] Gültekin, K.; Cackett, E. M.; King, A. L.; Miller, J. M. \& Pinkney, J 2014, ApJL, 788, L22

[28] Hughes, S. A. 2002, MNRAS, 331, 805

[29] Jardel, J. R., \& Gebhardt, K. 2012, ApJ, 746, 89

[30] Johnson, J. L., Whalen, D. J., Agarwal, B., Paardekooper, J.-P., \& Khochfar, S. 2014, MNRAS, 445, 686

[31] Kaaret, P.; Corbel, S.; Prestwich, A. H. \& Zezas, A. 2003, Science, 299, 365

[32] Kim, J.-h.; Wise, J. H.; Alvarez, M. A. \& Abel, T. 2011, ApJ, 738, 54

[33] Kormendy, J. \& Richstone, D. 1995, ARA\&A, 33, 581

[34] Kormendy, J., \& Ho, L. C. 2013, ARA\&A, 51, 511

[35] Liu, J.-F. \& Bregman, J. N. 2005, ApJS, 157, 59

[36] Lodato, G. \& Natarajan, P. 2006, MNRAS, 371, 1813

[37] Lora, V., Sánchez-Salcedo, F. J., Raga, A. C., \& Esquivel, A. 2009, ApJL, 699, L113

[38] Lützgendorf, N., Kissler-Patig, M., Noyola, E., et al. 2011, A\&A, 533, AA36

[39] Magorrian, J., Tremaine, S., Richstone, D., et al. 1998, AJ, 115, 2285

[40] Mapelli, M., Colpi, M., \& Zampieri, L. 2009, MNRAS, 395, L71

[41] Marleau, F. R., Clancy, D., Bianconi, M., \& Habas, R. 2014, arXiv:1411.3844

[42] McConnell, N. J. \& Ma, C.-P. 2013, ApJ, 764, 184

[43] McKernan, B., Ford, K. E. S., Lyra, W., et al. 2011, MNRAS, 417, L103

[44] Merloni, A.; Heinz, S. \& di Matteo, T. 2003, MNRAS, 345, 1057

[45] Mezcua, M., \& Lobanov, A. P. 2011, Astronomische Nachrichten, 332, 379

[46] Mezcua, M., Farrell, S. A., Gladstone, J. C., \& Lobanov, A. P. 2013a, MNRAS, 436, 1546 
[47] Mezcua, M., Lobanov, A. P., \& Martí-Vidal, I. 2013b, MNRAS, 436, 2454

[48] Mezcua, M., Roberts, T. P., Sutton, A. D., \& Lobanov, A. P. 2013c, MNRAS, 436, 3128

[49] Mezcua, M., Lobanov, A. P., Mediavilla, E., \& Karouzos, M. 2014a, ApJ, 784, 16

[50] Mezcua, M., Fabbiano, G., Gladstone, J. C., Farrell, S. A., \& Soria, R. 2014b, ApJ, 785, 121

[51] Mezcua, M., Roberts, T.P., Lobanov, A.P. Lobanov, \& Sutton, A.D. 2015, MNRAS, 448, 1893

[52] Mortlock, D. J.; Warren, S. J.; Venemans, B. P., et al. 2011, Nature, 474, 616

[53] Motch, C.; Pakull, M. W.; Soria, R.; Grisé, F. \& Pietrzyński, G. 2014, Nature, 514, 198

[54] Nemmen, R. S.; Georganopoulos, M.; Guiriec, S.; Meyer, E. T.; Gehrels, N. \& Sambruna, R. M. 2012, Science, 338, 1445

[55] Neumayer, N., \& Walcher, C. J. 2012, Advances in Astronomy, 2012, 709038

[56] Nyland, K.; Marvil, J.; Wrobel, J. M.; Young, L. M. \& Zauderer, B. A. 2012, ApJ, 753, 103

[57] Paragi, Z., Frey, S., Kaaret, P., et al. 2014, ApJ, 791, 2

[58] Pasham, D. R.; Strohmayer, T. E. \& Mushotzky, R. F. 2014, Nature, 513, 74

[59] Pérez-Ramírez, D.; Mezcua, M.; Leon, S. \& Caballero-García, M. D. 2011, Astronomische Nachrichten, 332, 384

[60] Peterson, B. M., Bentz, M. C., Desroches, L.-B., et al. 2005, ApJ, 632, 799

[61] Portegies Zwart, S. F.; Baumgardt, H.; Hut, P.; Makino, J. \& McMillan, S. L. W. 2004, Nature, 428, 724

[62] Poutanen, J.; Lipunova, G.; Fabrika, S.; Butkevich, A. G. \& Abolmasov, P. 2007, 377, 1187

[63] Reines, A. E.; Sivakoff, G. R.; Johnson, K. E. \& Brogan, C. L. 2011, Nature, 470, 66

[64] Reines, A. E.; Greene, J. E. \& Geha, M. 2013, ApJ, 775, 116

[65] Reines, A. E., Plotkin, R. M., Russell, T. D., et al. 2014, ApJL, 787, L30

[66] Remillard, R. A. \& McClintock, J. E. 2006, ARA\&A, 44, 49

[67] Ricotti, M., \& Ostriker, J. P. 2004, MNRAS, 350, 539

[68] Sánchez-Sutil, J. R.; Muñoz-Arjonilla, A. J.; Martí, J.; Garrido, J. L.; Pérez-Ramírez, D. \& Luque-Escamilla, P. 2006, A\&A, 452, 739

[69] Satyapal, S., Vega, D., Dudik, R. P., Abel, N. P., \& Heckman, T. 2008, ApJ, 677, 926

[70] Satyapal, S., Böker, T., Mcalpine, W., et al. 2009, ApJ, 704, 439

[71] Schramm, M.; Silverman, J. D.; Greene, J. E., et al. 2013, ApJ, 773, 150

[72] Secrest, N. J., Satyapal, S., Moran, S. M., et al. 2013, ApJ, 777, 139

[73] Seth, A. C., Cappellari, M., Neumayer, N., et al. 2010, ApJ, 714, 713

[74] Seth, A. C.; van den Bosch, R.; Mieske, S., et al. 2014, Nature, 513, 398

[75] Soria, R.; Long, K. S.; Blair, W. P., et al. 2014, Science, 343, 1330

[76] Strader, J., Chomiuk, L., Maccarone, T. J., et al. 2012, ApJL, 750, LL27

[77] Sutton, A. D.; Roberts, T. P.; Walton, D. J.; Gladstone, J. C. \& Scott, A. E. 2012, MNRAS, 423, 1154 
[78] Swartz, D. A.; Ghosh, K. K.; Tennant, A. F. \& Wu, K. 2004, ApJS, 154, 519

[79] Terashima, Y. \& Wilson, A. S. 2003, ApJ, 583, 145

[80] Terashima, Y., Kamizasa, N., Awaki, H., Kubota, A., \& Ueda, Y. 2012, ApJ, 752, 154

[81] Thim, F., Hoessel, J. G., Saha, A., et al. 2004, AJ, 127, 2322

[82] Thornton, C. E.; Barth, A. J.; Ho, L. C.; Rutledge, R. E. \& Greene, J. E. 2008, ApJ, 686, 892

[83] Valluri, M., Ferrarese, L., Merritt, D., \& Joseph, C. L. 2005, ApJ, 628, 137

[84] van der Marel, R. P., \& Anderson, J. 2010, ApJ, 710, 1063

[85] Volonteri, M. 2010, A\&A Rev., 18, 279

[86] Volonteri, M. \& Silk, J. 2014, arXiv: 1401.3513

[87] Webb, N.; Cseh, D.; Lenc, E., et al. 2012, Science, 337, 554

[88] Weiler, K.W., Panagia, N., Montes, M.J., Sramek, R.A., 2002, ARA\&A, 40, 38

[89] Wolter, A., Rushton, A. P., Mezcua, M., et al. 2014, arXiv:1412.5643

[90] Wrobel, J. M. \& Ho, L. C. 2006, ApJL, 646, L95

[91] Wrobel, J. M.; Greene, J. E.; Ho, L. C. \& Ulvestad, J. S. 2008, ApJ, 686, 838

[92] Wrobel, J. M.; Greene, J. E.; Ho, L. C. 2011, AJ, 142, 113

[93] Yuan, W.; Zhou, H.; Dou, L.; Dong, X.-B.; Fan, X. \& Wang, T.-G. 2014, ApJ, 782, 55

[94] Zampieri, L. \& Roberts, T. P. 2009, MNRAS, 400, 677 